\title{
CAPITAL SOCIAL, NEOLIBERALISMO Y CULTURA DE AUTOGESTIÓN PARA EL DESARROLLO
}

\author{
María Guadalupe Ortiz Gómez*
}

RESUMENः El capital social es un concepto que ha alcanzado una enorme relevancia en dos ámbitos: $a$ ) la investigación social, y $b$ ) el diseño de políticas de desarrollo. Sin embargo, su uso es controversial. En primer lugar, no representa una categoría nueva de análisis, ya que su campo de estudio constituye el mismo núcleo del objeto de la sociología. En segundo lugar, ciertas perspectivas sobre el capital social son funcionales para el modelo neoliberal. Finalmente, el uso que se le ha dado en el diseño de políticas de desarrollo tiende a ser problemático para la dinámica de las relaciones sociales en comunidades intervenidas bajo esta perspectiva. Este trabajo hace un análisis del concepto y su relación con las políticas neoliberales de desarrollo. Se plantea que dicha noción forma parte de una estrategia de promoción de una cultura de autogestión para el desarrollo que es funcional para el modelo neoliberal.

Palabras Clave capital social, neoliberalismo, cultura de autogestión para el desarrollo, políticas de desarrollo neoliberal.

\footnotetext{
* Posdoctorante en el Centro de Investigaciones Interdisciplinarias en Ciencias y Humanidades de la Universidad Nacional Autónoma de México, México.
} 
ABSTRACT: Social capital is a concept that has attained great relevance in two areas: a) social research, and b) the design of development policies. However, its use is controversial. First, it does not represent a new category of analysis, given that this field of study emerges from the nucleus as sociology. Second, some perspectives regarding social capital are enabling of the neoliberal model. Further, the use to which it has been applied in the design of development policies tends to be problematic for the dynamic of social relations in relevant communities under this perspective. This work analyzes the concept and its relationship with neoliberal development policies. It suggests that the idea is one part of a strategy to promote a culture of self-sufficiency in development that enables the neoliberal model.

KEY WORDS: social capital, neoliberalism, culture of self-sufficiency in development, neoliberal development policies. 


\section{INTRODUCCIÓN}

pesar de que el concepto de neoliberalismo está sujeto a complejas
discusiones en diferentes campos (filosófico, político, económico), ${ }^{1}$ y
que éste ha pasado por varias etapas en su implementación como proyecto de desarrollo (Ezcurra, 1998), su esencia sigue siendo la misma. Ésta implica una redefinición de los papeles del Estado, el mercado y la sociedad. Es un modelo que plantea la no intervención del Estado en la distribución de la riqueza. Amparados en la creencia de que la libre competencia asumirá ese papel, se sostiene que el Estado debe reducir a un mínimo su gasto social, lo que a su vez requiere de una ciudadanía más participativa y organizada que sea capaz de asumir la responsabilidad de su propio desarrollo (Ortiz, 2010 y 2013a). En este contexto, los organismos internacionales como el Banco Mundial (Bм) y el Banco Interamericano de Desarrollo (BID) promueven políticas desde las cuales se fomenta un nuevo papel ciudadano.

En un trabajo sobre políticas dirigidas a pueblos indígenas de México y Chile (Ortiz, 2010) se sostiene que existe un conjunto de ideas que son promovidas a través de los programas de financiamiento de desarrollo en localidades pobres de América Latina. Dichas ideas son coherentes con la necesidad del modelo neoliberal de contar con ciudadanos capaces de procurarse y gestionar sus propias opciones de desarrollo. Lo que incluye, a la vez, la adquisición de capacidades que les permitan resolver problemas que en el modelo de Estado de bienestar ${ }^{2}$ se consideraban responsabilidad de éste. En tal sentido he propuesto el término de cultura de la autogestión para el desarrollo (CAD) a fin de hacer referencia a dicho conjunto de ideas (Ortiz, 2010).

En Chile, Paley (2001) observó un fenómeno interesante. Cuando Patricio Aylwin asumió la presidencia (1990), después de 17 años de dictadura, había un alto grado de entusiasmo ciudadano, que se reflejaba en la disposición por participar en la construcción del nuevo proyecto

${ }^{1}$ Incluso el cultural (Ortiz, 2010 y 2013a).

2 Algunos autores argumentan que el modelo de Estado de bienestar nunca ha existido en América Latina, más bien parece suceder lo que podríamos llamar Estado de «malestar» (Assies, Calderón y Salman, 2001). 
de país. Sin embargo, la participación que el gobierno promovía se limitaba a la solución de problemas en los barrios, como la reparación de calles, lámparas, trabajos de limpieza, etcétera. No se alentaba, ni se veía con buenos ojos, la participación en relación a la toma de decisiones políticas. La autora sostiene que el discurso de participación ciudadana cumple entonces con dos objetivos: permitir a los gobiernos avanzar en sus agendas de reformas neoliberales, a la vez que justifica el retiro del Estado en cuanto a responsabilidad social.

En este sentido podemos argumentar que uno de los ejes del modelo de desarrollo neoliberal es la formación de ciudadanos participativos, solidarios, organizados y con el suficiente capital humano que les permita hacerse cargo de sí mismos (una idea central de la CAD). Ello, evidentemente, enmarcado de forma muy clara en los límites impuestos por los gobiernos nacionales y los organismos internacionales.

Una de las nociones más importantes en el discurso de la CAD es el llamado capital social. Los estudios de Putnam sostienen que existe una estrecha relación entre capital social y desarrollo económico. Tales ideas han sido sumamente controversiales, sin embargo constituyen uno de los sustentos principales de las políticas de desarrollo para países pobres. En este trabajo se presenta un análisis y problematización del concepto de capital social, asimismo se reflexiona sobre la forma en que es utilizado a favor del modelo neoliberal. El trabajo se divide en tres apartados. El primero tiene como objetivo presentar el contexto en el que se sitúa el análisis aquí desarrollado sobre el capital social. En el segundo se hace una revisión de las principales perspectivas desde las que se ha abordado el capital social. Finalmente, en el tercero se presentan una serie de reflexiones en torno al concepto, su relación con la CAD y las conclusiones finales.

\section{ENMARCANDO EL ANÁLISIS SOBRE EL CAPITAL SOCIAL}

El concepto de capital social ha cobrado una relevancia incuestionable en las últimas décadas (Durston, 2002; Portes, 1998; Ramírez, 2005; Putnam y Gross, 2003; Millán y Gordon, 2004). 
A pesar de ser controversial, la relevancia que tiene dicho concepto es grande, ya que las políticas públicas de desarrollo de las últimas décadas, a nivel global, lo han incluido como uno de sus componentes principales. Una evidencia de lo anterior es que el вм ha posicionado al capital social como un eje transversal en sus programas de intervención en grupos sociales marginados (Ramírez, 2005: 22). Se parte de la idea de que es un recurso vital para el desarrollo y que es necesario invertir en su conservación y fortalecimiento. Estas ideas provienen, principalmente, de los planteamientos de Putnam, quien al comparar dos regiones de Italia (el norte con el sur), concluye que existe una relación directa entre desarrollo, democracia y capital social. En este sentido, la región norte de Italia es más próspera que la del sur, debido a que la primera cuenta con un capital social más rico (Portes, 1998; Sisiiäinem, 2000: 3 y Kliksberg, 1999: 87).

A pesar de que la postura de Putnam ha sido ampliamente criticada, sirvió para poner de relieve la relación existente entre cultura, ${ }^{3}$ capital social y desarrollo (Portes, 1998; ver también Kliksberg, 1999). Por ello se ha llegado a concebir al capital social como el remedio para todos los males de las sociedades contemporáneas (Portes, 1998).

Si se analizan estas tendencias dentro del marco de la aplicación del modelo neoliberal, se observa que el concepto presenta una funcionalidad muy clara para éste (Ramírez, 2005, Sisiiäinem, 2000, Ortiz, 2010). Como han argumentado diferentes autores (Assies, 1999; Grimson, 2007; Ortiz, 2010 y 2013a), el neoliberalismo es un proyecto donde el componente cultural es importante. Al modificarse las funciones del Estado ante los ciudadanos, estos últimos deben adquirir nuevas responsabilidades y prácticas que doten de funcionalidad a las políticas neoliberales. Se trata de modificar la visión que se tiene sobre el papel del Estado, el mercado y la sociedad. ${ }^{4}$

\footnotetext{
${ }^{3}$ Para este autor, específicamente la cultura cívica.

${ }^{4}$ Se reconoce que las relaciones entre estos tres componentes son estrechas y que no se trata de bloques con fronteras definidas, ya que algunos ciudadanos son parte también del aparato estatal y a la vez agentes del mercado, etcétera; sin embargo es útil hacer esta separación para los fines
} 
Con base en conceptos sociológicos y antropológicos se modificó la estrategia de desarrollo pasando de la inversión en recursos materiales (que buscaba resolver los problemas en lo inmediato) a la inversión en capital humano (que busca la resolución de los problemas a largo plazo y de una manera sostenida) (Montufar, 2001). Más tarde se incorporó el concepto de capital social, ya que el capital humano no garantizaba la sustentabilidad de un proyecto de desarrollo. Se necesitan grupos sociales con capacidad de autogestión, ${ }^{5}$ por lo que es importante fortalecer sus capacidades organizativas, de solidaridad, de corresponsabilidad, etcétera. Con ello se imprime al comunitarismo un sentido pragmático (Hale, 2003; Siisiäinen, 2000).

\section{CAPITAL SOCIAL, SURGIMIENTO Y DESARROLLO}

Lo que actualmente es conocido como capital social se encuentra en el centro mismo del objeto de estudio de la sociología. Portes nos advierte que el tema no es nuevo, se remonta a la idea de Durkheim de que la vida social del grupo es un antídoto contra la anomia, y a la distinción de Marx entre la clase atomizada en sí y la clase movilizada para sí.

Bevort (2007) argumenta que los orígenes de este concepto pueden encontrarse en perspectivas sociológicas como la de Alexis de Tocqueville, quien sostiene que la forma en que los hombres se relacionan entre sí es importante para la construcción de una sociedad desarrollada. Explica que «el amor juicioso de ellos mismos les conduce sin cesar a ayudarse entre sí y los predispone a sacrificar con gusto por el bien del Estado una parte de su tiempo y sus riquezas» (Tocqueville citado en Bevort, 2007: 2). Weber también habló sobre el tema en sus estudios sobre religión. Los miembros de un grupo tienen que cumplir ciertas normas

de análisis que aquí se persiguen. Durston (2002) se refiere a esos espacios de interacción como interfaces entre las diferentes esferas.

${ }_{5}^{5}$ Autogestión entendida como capacidad para solucionar problemas, no para la toma de decisiones (Ortiz, 2013a). 
para ser admitidos. A diferencia de Tocqueville, Weber ve al hecho asociativo como prácticas religiosas que se van secularizando (Bevort, 2007:3).

A pesar de que no aporta nada nuevo (Portes, 1998: 3; Bevort, 2007: $1)$, su análisis es necesario, ya que se trata de un elemento clave dentro de las políticas de desarrollo de las últimas décadas. La pregunta sociológica de fondo se podría formular de la siguiente manera: ¿qué hace que un grupo humano tenga capacidad para vivir y actuar en conjunto, para ponerse de acuerdo sobre una orientación común, un proyecto común o un destino común? (Bevort, 2007:4). Si existen múltiples desarrollos teóricos para dar respuesta, debemos reflexionar por qué el capital social se ha logrado posicionar como un concepto clave, no sólo en el ámbito académico, sino también en relación al diseño de políticas públicas.

Enseguida abordaremos tres visiones sobre el capital social de autores que son considerados clásicos: Putnam, Coleman y Bourdieu. ${ }^{6}$ Tanto las corrientes académicas como los intereses de investigación son distintos en los tres casos. En Putnam el interés principal es descifrar cuáles son las precondiciones para el desarrollo de instituciones fuertes, responsables y democráticas, así como para la prosperidad económica (Siisiäinem, 2000: 3). Se enfoca en el estudio de los sistemas políticos y la cultura cívica; Coleman se interesa en el análisis del comportamiento económico de los individuos; mientras que Bourdieu trata de explicar cómo se reproducen las estructuras sociales y las diferencias de clase mediante su teoría de los capitales.

\section{La perspectiva de Putnam}

Putnam no es el primer autor que habló de capital social. Éste tomó el concepto de Coleman, pero se lo apropió de una forma particular, seleccionando

${ }^{6}$ Millán y Gordon (2004) postulan como clásicos a Putnam, Coleman y Lin. El orden en que se presentan responde al nivel de complejidad que imprimen los autores al concepto. 
algunos elementos y añadiendo otros (Ramírez, 2005: 30). Afirma que el capital social se compone esencialmente de confianza, normas de reciprocidad y redes de compromiso cívico (Ramírez, 2005: 30; Siisiäinem, 2000). La argumentación de Putnam proviene de una visión funcionalista de la sociedad, donde el conflicto es percibido como una patología. Para Siisiäinem, Putnam cae, de cierto modo, en la ingenuidad debido a su idea de que puede existir una confianza generalizada que lleve a una convivencia social exenta de conflicto (Siisiäinem, 2000: 3). La clave de una sociedad desarrollada es, desde esta perspectiva, el fortalecimiento de las redes sociales a través de la confianza, la reciprocidad, el compromiso con la comunidad, etcétera. Contrariamente a quienes afirman que el asociacionismo y la autoorganización bloquean la eficacia gubernamental y el desarrollo económico, Putnam sostiene que son las redes de compromiso cívico las que promueven el crecimiento económico y la estabilidad política (Ramírez, 2005: 31; ver también Putnam y Gross, 2003).

Las reservas (stocks) de capital social, tales como la confianza, las normas y las redes, tienden a ser autorreforzantes y acumulativas. Círculos virtuosos resultan en un equilibrio social con altos niveles de cooperación, confianza, reciprocidad, compromiso cívico, y bienestar colectivo. Estos rasgos definen a la comunidad cívica. De manera inversa, la ausencia de estos rasgos en la comunidad «acívica»son también autorreforzantes (Putnam citado en Ramírez, 2005: 30).

Putnam nos dice que «la idea central de la teoría del capital social es sumamente sencilla: las redes sociales importan. Las redes poseen valor, ante todo, para quienes se hallan en ellas. Utilizando el lenguaje de la microeconomía, las redes producen beneficios privados o internos» (Putnam y Gross, 2003: 13). Woolcock y Narayan sostienen que:

La idea básica del capital social es que la familia, los amigos y los compañeros de una persona constituyen un valor importante al qué recurrir en una crisis, del qué disfrutar por sí mismo y del qué servirse para conseguir ventajas materiales. Además, esto, que es cierto para los individuos, vale también para 
los grupos. Las comunidades con recursos variados de redes sociales y asociaciones cívicas se encuentran en una posición más sólida para hacer frente a la pobreza y la vulnerabilidad, resolver disputas y sacar partido a oportunidades nuevas (Woolcock y Narayan, citados en Putnam y Gross, 2003: 12).

Las redes sociales, en este sentido, tienen un valor material que se puede traducir en beneficios económicos. Incluso se han realizado estudios para calcular el valor monetario de una red, ya que los ingresos de una persona están determinados por el alcance de sus relaciones sociales (Putnam y Gross, 2003: 13). Este tipo de aproximaciones al capital social se antojan simplistas, ya que lejos de hacer un análisis situado en un contexto social más amplio, establecen una relación directa entre capital social e ingresos económicos, sin considerar elementos importantes como la estratificación social (división de clases) y las relaciones de poder. ${ }^{7}$

Después de recibir serias críticas, Putnam reconoce que el capital social puede tener un lado oscuro. Después de todo, las organizaciones criminales cuentan con un importante capital social que les permite operar (Portes, 1998: 15; Ramírez, 2005: 32). De igual forma, nos dice que:

Las redes y las normas podrían beneficiar, por ejemplo, a quienes están bien instalados, en detrimento de quienes no lo están. El capital social podría hallarse especialmente extendido entre grupos de personas que ya disfrutan de ventajas, aumentando así las desigualdades políticas y económicas entre esos grupos y los pobres en capital social (Putnam y Gross, 1003: 16).

Esta última observación coincide con la perspectiva de Bourdieu, sin embargo, en Putnam no existe un desarrollo teórico que permita explicar este fenómeno, ya que ha dejado fuera el análisis de la estratificación social.

7 Cabe señalar que Coleman sí incluyó las relaciones de autoridad y jerarquía (como las que estructuran a las organizaciones lucrativas) como formas de capital social, mientras que Putnam tuvo mucho cuidado en dejarlas fuera (Ramírez, 2005: 31). 
La mayoría de las críticas a Putnam se ha centrado en la importancia de las asociaciones y de la interacción informal en la construcción de la confianza y la cooperación. No hay una explicación acerca de cómo se generalizan e institucionalizan las normas de reciprocidad y los valores de confianza. Respecto de la capacidad de producción de normas de reciprocidad generalizada de las asociaciones, Levi (1996) pone en duda que la pertenencia a un tipo de asociación lleve a superar problemas de free riding en otra, dado que hay diferencias entre los distintos tipos de clubes sociales entre sí y respecto de las organizaciones orientadas a la acción política. También indica que Putnam no ha hecho explícitos los mecanismos por medio de los cuales la pertenencia a asociaciones o clubes conduce a un alto nivel de compromiso cívico y de política democrática, señalamiento en el que coinciden varios autores (Millán y Gordón, 2004: 736).

Otros críticos han llamado la atención sobre el hecho de que la confianza generalizada se asocia con niveles bajos de desigualdad en los ingresos, sin embargo, esta relación debe cuestionarse ya que suele ser mucho más compleja (Siisiäimen, 2000). En lo que se refiere al desempeño gubernamental, se ha criticado que Putnam busque la explicación de las diferencias entre el norte y el sur de Italia en el compromiso cívico de los ciudadanos, y no en la estructura del sistema político. En estrecha relación con esta crítica, se ha señalado el hecho de que en la formulación de Putnam, el Estado es externo al modelo explicativo (Millán y Gordón, 2004: 737).

La falta mayor de Putnam es dejar fuera de su análisis a la estratificación social y las relaciones de poder al interior de los grupos. Ello le ha impedido dar consistencia a su propuesta teórica, ya que no es capaz de dar cuenta de una serie de fenómenos sociales relacionados con la desigualdad social. Lo anterior conduce, igualmente, a interpretaciones erróneas, ya que al postular al capital social como la fuente primaria del desarrollo, se pierden de vista las condiciones estructurales. Esta interpretación se presta a responsabilizar de la pobreza a la falta de capital social de algunos grupos, lo cual resulta extremadamente reduccionista (Siisiämen, 2000). 


\section{La perspectiva de Coleman}

Una de las grandes diferencias entre Coleman y Putnam es que el primero se inscribe en una teoría sociológica de largo alcance, mientras que el segundo se mueve en una propuesta teórica de alcance medio, ya que su interés específico es el estudio de los sistemas democráticos (Millán y Gordon, 2004: 74). Coleman tiene el mérito innegable de introducir y dar visibilidad al concepto en la sociología estadounidense, resaltando su importancia para la adquisición de capital humano e identificando algunos de los mecanismos a través de los cuales es generado (Portes, 1998: 6; Ramírez, 2005; Millán y Gordon, 2004; Bevort, 2007). Percibe al capital social como una herramienta que ayuda a su interés teórico más amplio de importar el principio económico de la acción racional para su uso en el análisis del propio sistema social, incluido pero no limitado al análisis del sistema económico, y hacerlo sin descartar en el proceso a la organización social (Coleman, citado en Ramírez, 2005: 26).

El capital social representa una doble función explicativa: permite dar cuenta de diferentes resultados en el plano del actor individual y, a la vez, hacer transiciones micro-macro sin tener que describir los detalles de la estructura a través de la cual ocurre. La definición de Coleman de capital social subraya ambas dimensiones. En su libro Foundations of Social Theory (1990), este autor define el capital social como recursos de los individuos que facilitan su acción dentro de estructuras en las que se encuentran. El capital social es definido por su función, no es una entidad única sino una variedad de diferentes entidades, con dos elementos en común: todas ellas consisten de algún aspecto de las estructuras sociales y facilitan la realización de ciertas acciones para los actores -individuales o colectivos - dentro de la estructura (Coleman citado en Bevort, 2007:5; Portes, 1998: 5; Ramírez, 2005: 26). Coleman señala que «los grupos constituyen un recurso que ayuda a pasar de la protesta individual a la revuelta organizada» (Coleman citado en Ramírez, 2005: 27). Se inscribe explícitamente en la corriente de la elección racional, al 
tiempo que subraya que el capital social es tanto propiedad de los actores como la caracterización de una estructura de relaciones.

El rasgo distintivo del capital social es su carácter funcional, que es, de alguna manera, instrumental. Al igual que otras formas de capital, el capital social es productivo y hace posible el logro de ciertos fines que serían inalcanzables en su ausencia. Por ejemplo, un grupo cuyos miembros confían ampliamente unos en otros está en capacidad de lograr mucho más en comparación con un grupo donde no existe la confianza (Millán y Gordon, 2004: 714). En este sentido, en cualquier aspecto de la estructura social que contribuya a la realización de los fines del actor, existe capital social. Todas las relaciones y estructuras sociales facilitan alguna forma de capital social (Ramírez, 2005: 27). A través de las redes de relaciones se crean obligaciones y expectativas recíprocas que dependen del grado de confianza mutua que exista dentro de una estructura social dada. Se forman canales informales de comunicación capaces de hacer circular eficazmente las informaciones $y$, finalmente, hacer surgir normas y la seguridad de que éstas se apliquen (Bevort, 2007: 5-6, ver también Portes, 1998).

Una de las características del concepto de Coleman es que piensa que el capital social puede ser creado, mantenido o destruido. Este autor argumentó que la propiedad de «cierre» (closure) $)^{8}$ de las relaciones sociales, la estabilidad de la estructura social, así como la ideología, podían ser los principales factores que influyan en esta dinámica (Ramírez, 2005: 28). En general, el capital social podría verse afectado, si no se le da mantenimiento constante, puede depreciarse. Según Coleman, como sucede con otros tipos de capitales: «las relaciones sociales mueren si no se mantienen; las expectativas y obligaciones se marchitan con el paso del tiempo y las normas dependen de la comunicación regular» (Coleman citado en Ramírez, 2005: 28).

${ }^{8}$ La idea de clausura (closure) en las relaciones refiere en términos generales tanto al surgimiento y permanencia de normas efectivas como a la posibilidad de mantener vigentes elementos externos de cohesión (Portes, 1998: 6; Millán y Gordon, 2004: 719). 
Resultan polémicas sus ideas acerca de que habría que considerar como destructivo del capital social a todo aquello que haga a las personas menos dependientes unas de otras. Por ejemplo, el tema de la riqueza y de la asistencia gubernamental en caso de necesidad, ya que ello hace que las personas no pidan ayuda unas a otras. Ello es coherente con las ideas surgidas del Consenso de Washington, mediante las cuales se argumenta que si bien el libre mercado no es perfecto, la intervención estatal es más perjudicial (Ramírez, 2005: 28). La idea de que la riqueza destruye el capital social contradice ampliamente los postulados de Putnam, ya que para éste la relación entre capital social y prosperidad económica es positiva.

Otra característica de la propuesta de Coleman es que considera que el capital social es a la vez una propiedad individual y colectiva, a diferencia de Putnam, quien sostiene que es un bien colectivo (Millán y Gordon, 2004). El capital social como propiedad individual tiene que ver con el grado de integración social de una persona y con la capacidad para hacer una red de contactos sociales. Implica relaciones, expectativas de reciprocidad y comportamientos confiables, y mejora la eficacia privada. Es un bien colectivo si, por ejemplo, todos los miembros de un vecindario siguen normas tácitas de solidaridad con los demás y de no agresión, los niños podrán caminar a la escuela con seguridad y el capital social estará produciendo orden público (Coleman citado en Kliksberg, 1999: 87).

Una de las críticas que se pueden hacer a esta perspectiva es la visión instrumentalista sobre el capital social. Ésta obedece al hecho de que Coleman está tratando de explicarse la dinámica de los intercambios económicos, sin dejar de lado el análisis de las relaciones sociales. La analogía del capital social con el capital económico, o físico, es útil para fines analíticos, pero no se debe perder de vista que no existe equivalencia posible entre capital económico y social. No existe una unidad de medida que permita calcular el valor económico del capital social. A pesar de ello se han hecho esfuerzos como el mencionado por Putnam.

A pesar de sus posibles limitaciones, Coleman logró poner en la agenda de discusión algunas cuestiones que han sido útiles para entender 
la manera en que se distribuyen los diferentes tipos de capitales. Sin embargo, quien logra analizar de una forma más refinada esta cuestión es, sin lugar a dudas, Bourdieu (Portes, 1998).

\section{La perspectiva de Bourdieu}

Aún en vida, a Pierre Bourdieu se le considera un clásico de la teoría social (Giménez, 2002). Él mismo caracterizó a su paradigma como «constructivismo estructuralista». ${ }^{9}$ Se trata de aprehender las realidades sociales como construcciones históricas y cotidianas de actores individuales y colectivos, construcciones que tienden a substraerse a la voluntad clara y al control de estos mismos actores (Giménez, 2002). La tesis fundamental de la teoría de Bourdieu es que las realidades sociales son a la vez objetivadas e interiorizadas. Es decir, por una parte, remiten a mundos objetivados (estructuras: reglas, instituciones, etcétera) exteriores a los agentes, que funcionan a la vez como condiciones limitantes y como puntos de apoyo para la acción y, por otra, se inscriben en mundos subjetivos e interiorizados, constituidos principalmente por formas de sensibilidad, percepción, representación y conocimiento (Giménez, 2002).

Para el estudio de una sociedad, entonces, es necesario entender las relaciones entre estructuras y sujetos; a la vez que para entender tal relación debemos conocer el contexto más amplio en el que se da, es decir, un espacio donde los sujetos tienen diferentes posiciones y desde donde realizan esta operación de interiorización y reproducción de la realidad social. Es importante destacar que desde la perspectiva de Bourdieu las posiciones que ocupan los sujetos implican una diferenciación, lo que él ha llamado la distinción. La posición que se ocupa en la estructura

\footnotetext{
9 La problemática constructivista no constituye una nueva escuela ni una nueva corriente homogénea desde el punto de vista teórico o metodológico. Se trata más bien de un espacio de problemas y de cuestiones en torno a los cuales giran los trabajos de un número significativo de investigadores, pese a la diversidad de sus tradiciones y de sus itinerarios intelectuales (Corcuff citado en Giménez, 2002).
} 
depende de la cantidad de capitales que se posee; en este sentido estamos hablando de que las sociedades se componen de estratos diferenciados.

Para Bourdieu el espacio social es un sistema de posiciones sociales donde se definen las unas en relación con las otras (por ejemplo, autoridad/súbdito; jefe/subordinado; patrón/empleado; hombre/mujer; rico/ pobre; distinguido/popular). El «valor» de una posición se mide por la distancia social que la separa de otras posiciones inferiores o superiores, lo que equivale a decir que el espacio social es, en definitiva, un sistema de diferencias sociales jerarquizadas ${ }^{10}$ (la distinción) en función de un sistema de lesgitimidades socialmente establecidas y reconocidas en un momento determinado. Y lo que se llama «orden social» no sería más que el sistema global de espacios sociales constituido por conjuntos de posiciones, a la vez vinculadas y contrapuestas entre sí por las distancias que las separan (Giménez, 2002).

En las sociedades modernas caracterizadas por un alto grado de diferenciación y complejidad, el espacio social se torna multidimensional y se presenta como un conjunto de campos relativamente autónomos, aunque articulados entre sí: campo económico, campo político, campo religioso, campo intelectual. Un campo, por lo tanto, es una esfera de la vida social que se ha ido autonomizando progresivamente a través de la historia en torno a cierto tipo de relaciones sociales, de intereses y de recursos propios, diferentes a los de otros campos (Giménez, 2002). Bourdieu introduce una analogía para explicar esta idea y nos presenta al campo como un espacio de juego relativamente autónomo, con objetivos propios a ser logrados, con jugadores compitiendo (a veces ferozmente) entre sí y empeñados en diferentes estrategias según su dotación de cartas y su capacidad de apuesta (capital), pero al mismo tiempo interesados en jugar porque creen en el juego y reconocen que vale la pena jugar (Bourdieu, 1992: 73). En cada momento, las relaciones de fuerza entre los jugadores definen la estructura del campo. Esto no es todo, los jugadores pueden jugar para aumentar o conservar su capital, sus fichas, es decir,

\footnotetext{
${ }^{10}$ Esta idea es marginal en la perspectiva de Putnam.
} 
de conformidad con las reglas tácitas del juego y con las necesidades de la reproducción del juego y de los intereses en juego; pero también pueden trabajar para transformar parcial o totalmente las reglas inmanentes del juego (Bourdieu, 1992: 75).

En sentido riguroso, el campo se define - como todo espacio social- como una red o una configuración de relaciones objetivas entre posiciones diferenciadas, socialmente definidas y en gran medida independientes de la existencia física de los agentes que las ocupan (...). Así, el agente que ocupa la posición de empleado o de patrón, de sub-oficial o de oficial superior, de padre o de hijo, de dirigente deportivo o de simple practicante, puede muy bien desaparecer físicamente sin que la posición deje de existir, quedando ésta disponible para otro agente según el principio: ha muerto el rey, ¡viva el rey! (Giménez, 2002: 7).

La especificidad de cada campo está determinada por el tipo de recursos (o la combinación particular de tipos de recursos) que se moviliza y tiene curso en su ámbito. A pesar de su aparente diversidad, estos recursos pueden reagruparse en tres grandes categorías:

- Recursos de naturaleza económica (entre los que el dinero ocupa un lugar preeminente por su papel de equivalente universal).

- Recursos de naturaleza cultural (entre los cuales los diplomas escolares y universitarios han cobrado una importancia creciente).

- Recursos sociales consistentes en la capacidad de movilizar en provecho propio redes de relaciones sociales más o menos extensas, derivadas de la pertenencia a diferentes grupos o «clientelas» (Giménez, 2002).

A estos recursos podemos considerarlos como capitales. Las diferentes especies de capital están estrechamente vinculadas entre sí, y bajo ciertas condiciones pueden transformarse unas en otras. Bourdieu habla incluso de cierta «tasa de convertibilidad» entre los diferentes tipos de capital (Giménez, 2002). De esta forma, por ejemplo, el capital cultural puede 
traducirse en capital económico a través del ejercicio de una profesión avalada por un título universitario. ${ }^{11}$

Es importante señalar que el capital acumulado de modo específico en un determinado campo se distribuye ordinariamente de modo desigual entre los agentes según la posición ocupada. En efecto, lo que se observa en la realidad es una estructura de distribución del capital específico más o menos dispersa o concentrada según la historia del campo considerado y, por lo mismo, según la evolución de las luchas por la apropiación del capital (Siisiäinen, 2000). A partir de esto puede entenderse la relación entre capital y poder. La relación de fuerzas resultante de la desigual distribución del capital en cuestión es lo que define las posiciones dominantes y dominadas dentro de un campo y, por lo tanto, la capacidad de ejercer un poder $y$ una influencia sobre otros. Es decir, el hecho de disponer personalmente de bienes económicos y culturales es fuente de poder con respecto a los que los poseen en menor medida o simplemente carecen de ellos (Giménez, 2002). Por ello, Bourdieu parte de una perspectiva que considera al conflicto como parte de la dinámica social, y no como una patología, al estilo de Putnam.

No es casual que el tema de la educación le haya interesado fuertemente a Bourdieu, pues ve en él una clave importante para entender cómo se dan los intercambios de capitales y cómo las estructuras de las sociedades modernas tienden a perpetuar los estratos jerárquicos de clase. Se trata de una especie de círculo en donde los poseedores de algún tipo de capital tienen más oportunidad de tener acceso a los otros tipos. Por ello, en las sociedades desarrolladas, las clases medias le dan una gran importancia a la obtención de un título universitario. Bourdieu lo asemeja, de hecho, a la obtención de un título nobiliario.

El capital social no es un punto central en el análisis de Bourdieu, su abordaje le interesa sólo en relación a los otros tipos de capital. Ello le permite explicar la reproducción de las estructuras de clase. Introducir la noción de capital social hace posible entender por qué dos personas

${ }^{11}$ Dicha convertibilidad es compleja y la idea de poder calcular el valor del capital cultural o social es problemática. 
con cantidades equivalentes de capital económico o cultural, obtienen diferentes beneficios (Ramírez, 2005: 23).

En su trabajo sobre Las formas del capital (1985), Bourdieu argumenta que el capital social se constituye por la totalidad de los recursos potenciales o actuales asociados a la posesión de una red duradera de relaciones más o menos institucionalizadas de conocimiento y reconocimiento mutuos. Expresado de otra forma, se trata aquí de la totalidad de recursos basados en la pertenencia a un grupo (Bourdieu citado en Ramírez, 2005: 23).

Según Ramírez existen cuatro elementos esenciales que articulan la teoría sobre el capital social de Bourdieu, y que es necesario precisar para entender el alcance de su propuesta: la pertenencia a un grupo, la existencia de relaciones de intercambio material y simbólico que se dan en su interior, el grado de institucionalización y los recursos que posee dicho grupo (Ramírez, 2005: 23). En este sentido, a diferencia de los otros tipos de capital, el social requiere de una red duradera de relaciones sociales. La permanencia de estos grupos es posible gracias a los intercambios que se realizan entre los miembros. Se intercambian bienes materiales, pero también simbólicos. Dicho intercambio asume la forma de un toma y daca en apariencia desinteresado, gratuito y voluntario, pero que genera en realidad un cúmulo de obligaciones duraderas que «se apoyan bien sobre sentimientos subjetivos (de reconocimiento, respeto, amistad y demás), bien sobre garantías institucionales (derechos o pretensiones jurídicas)» (Bourdieu citado en Ramírez, 2005: 24) y que, junto con el «conocerse y reconocerse» mutuo, sirve para fijar los límites del grupo (Ramírez, 2005: 23-24).

A diferencia de Putnam, Bourdieu no considera capital social a las relaciones difusas. Cree que tiene que existir un mínimo de homogeneidad entre los miembros y el común reconocimiento a una cierta institucionalización. Bourdieu aduce que el capital social en posesión de un grupo tiende a concentrarse en manos de un individuo o unos pocos mediante «delegación institucionalizada», tanto más conforme el grupo sea más grande y menos poderoso (Ramírez, 2005: 24). 
Es importante remarcar que para este autor los diferentes tipos de capitales tienen una relación dialéctica. El volumen de capital social de un individuo depende no sólo de la red de conexiones que posea, sino de la cantidad de capital económico, cultural o simbólico, poseído por aquellos con quienes está relacionado. El volumen de capital social en posesión de una red, a final de cuentas, dependerá en gran medida del volumen de capital económico y cultural de ese grupo. Esto ocasionaría lógicamente una distribución desigual del capital social entre los grupos o redes que conforman la sociedad (Ramírez, 2005: 25).

Desde esta perspectiva, la relación entre desarrollo (económico y político) y capital social de un grupo social determinado no sería directa, como lo establece Putnam. Los grupos sociales no son homogéneos y se constituyen de estructuras jerárquicas, por tanto, el desarrollo (económico y cultural) dependerá de la dinámica que adquiere la distribución de capitales en el espacio social.

\section{REFLEXIONES EN TORNO AL CAPITAL SOCIAL Y CONCLUSIONES}

A pesar de la gran cantidad de trabajos y los diversos enfoques que existen sobre el capital social, es posible identificar algunas ideas generales. La primera de ellas es que las investigaciones basadas en este concepto han contribuido a empobrecer la discusión teórica al respecto (Portes, 1998). La mayoría se basa en el famoso estudio de Putnam sobre Italia o en los estudios de Coleman. Se limitan a ensayar estrategias metodológicas útiles para la corroboración de las ideas surgidas de estas perspectivas (Ramírez, 2005). Hasta hace algunos años no existían trabajos que se enfocaran en rastrear el surgimiento del concepto y los usos que se han hecho de él en las ciencias sociales. Portes nos ofrece un excelente artículo en el cual evidencia la superficialidad del enfoque de Putnam en relación a otros autores como Bourdieu y Coleman. En esa misma línea, Ramírez (2005) nos presenta un buen aná- 
lisis de tres perspectivas teóricas que considera clásicas, se trata de Bourdieu, Coleman y Putnam. ${ }^{12}$ De igual manera, el mismo Putnam hace su propio seguimiento del concepto (Putnam y Gross, 2003), en el cual llama la atención la escasa importancia que otorga a las ideas de Bourdieu.

Siisiäinem (2000) hace una comparación entre Putnam y Bourdieu y establece que existe una relación entre la perspectiva de Putnam y las estrategias para imponer una forma de globalización económica al estilo neoliberal. Argumenta, de la misma forma, que el concepto del capital social es útil para responsabilizar a los pobres mismos de su pobreza, ya que se deja fuera del análisis a las estructuras sociales que permiten una distribución desigual de los capitales. Por su parte, Ramírez nos muestra cómo el contenido del concepto se modifica de acuerdo con las corrientes o escuelas sociológicas de las que provienen sus autores. Para Bourdieu, el capital social es principalmente un mecanismo de diferenciación social y reproducción de las clases; mientras que Coleman lo concibe como el vínculo entre el actor y la estructura; finalmente, para Putnam el capital social es un recurso que posee la comunidad (Ramírez, 2005).

Desde mi punto de vista debemos preguntarnos por qué se ha tomado la perspectiva de Putnam como uno de los ejes orientadores en el diseño de las políticas de desarrollo a nivel global. Si existen versiones mucho más ricas en un sentido teórico, resulta interesante reflexionar sobre por qué una visión que parece simplista sobre el capital social es la que ha alcanzado mayor popularidad en los organismos que financian el desarrollo. Este hecho resulta coherente cuando lo situamos en el contexto de los planteamientos del modelo neoliberal. Resulta útil una perspectiva como la de Putnam en dos sentidos principales. El primero porque invisibiliza la distribución desigual de los capitales y el cómo esta misma dinámica tiende a perpetuar a grupos poseedores de mayor capital en posiciones de más alta jerarquía. La asociación automática

\footnotetext{
${ }^{12}$ En mi opinión, los primeros dos por sus aportes teóricos y el tercero más por la influencia de su trabajo en el diseño de políticas y por la promoción que el Banco Mundial le ha hecho a su perspectiva.
} 
que plantea Putnam entre capital social y prosperidad económica responsabiliza a los grupos sociales de sus condiciones económicas. Es decir, si un grupo no es próspero económicamente, ello se atribuye al hecho de que su capital social no es suficiente. De esta forma no habría otros componentes del conjunto social a quienes pudiera atribuírseles alguna responsabilidad (por ejemplo el Estado, los monopolios).

Debemos retomar algunos planteamientos del modelo neoliberal que nos permitirán entender por qué el capital social se ha posicionado como eje de las políticas de desarrollo. Como arriba se mencionó, existe una reformulación de los papeles del Estado, el mercado y la sociedad. Uno de los rasgos que nos interesa destacar de dicha reformulación es que el ente que se concibe como el encargado más eficiente de realizar una distribución «justa» de la riqueza es el mercado. Ya no es el Estado (como en su versión de bienestar) quien tiene que velar por una redistribución del ingreso que tienda a la equidad, por tanto la ética social de éste desaparece. Se disminuye el gasto social y la responsabilidad de generar oportunidades de desarrollo y de acceso a recursos recae en el propio ciudadano. En este sentido, desde hace varias décadas, se están haciendo diferentes esfuerzos por parte de organismos internacionales y gobiernos nacionales para promover ideas, valores e identidades acordes al nuevo papel del ciudadano. Como ya mencioné arriba, se trata de una cultura de autogestión para el desarrollo (CAD), misma que se compone de diferentes elementos, dentro de los cuales el capital social es uno de los más importantes (Ortiz, 2013a).

En resumen, se necesitan grupos sociales organizados que sean capaces de enfrentar las vicisitudes del libre mercado y a la vez llenar los huecos que dejó el Estado en relación al gasto social, 2001). En México podemos apreciar que mediante diferentes programas de política pública se ha promovido un discurso que critica las actitudes paternalistas de gobiernos anteriores a la vez que alienta a los ciudadanos para que cultiven su espíritu emprendedor. Recordemos a Vicente Fox cuando dijo que 
todos deberíamos tener «vocho y changarro», ${ }^{13}$ así como la creación del Instituto Nacional del Emprendedor en 2013, entre otras acciones. Estas ideas son coherentes con el papel que se plantea desde el modelo neoliberal para los ciudadanos, ya que se tiene la idea de que todos debemos desarrollar capacidades para insertarnos en el mercado de forma competitiva. De ahí que el capital social (junto con el capital humano) se promueva como un recurso vital para la formación de grupos competitivos.

Desde este panorama surgen varias cuestiones problemáticas. Una de las principales es que la dinámica de mercado tiende a favorecer a los grupos con mayores capitales (como lo argumenta Bourdieu). Los monopolios acaparan los mercados, lo que margina a la mayoría de pequeños emprendedores. La propuesta neoliberal sobre el papel ciudadano tiende a la homogeneización de los mismos. El ciudadano «autogestionado» forzosamente debe buscar su sitio en el mercado. Si consideramos que el desempleo es una de las características comunes que resultan de la aplicación de políticas neoliberales, se está presionando a los ciudadanos para que se conviertan en microempresarios. Pero no se toma en cuenta si tales ciudadanos tienen la vocación y las habilidades para serlo.

Por otro lado, existe una fuerte contradicción relacionada con las políticas que promueven el capital social como recurso de desarrollo en localidades empobrecidas de América Latina. Generalmente se promueve el capital social comunitario con la idea de fortalecer grupos con potencial emprendedor. Tal es el caso de los programas ofrecidos por la Comisión Nacional para el Desarrollo de los Pueblos Indígenas (CDI). Un ejemplo interesante en este sentido lo encontramos en la Isla de Yunuén, en el municipio de Pátzcuaro, Michoacán. En dicha localidad se construyeron cabañas para ofrecer servicios turísticos con la idea de diversificar sus fuentes de ingresos y frenar la alta migración que prevalece en la misma.

\footnotetext{
${ }^{13}$ En este sentido también podemos ver la participación de la iniciativa privada en México, específicamente del Consejo de la Comunicación con su campaña de «Pepe y Toño». A pesar de tener un carácter privado, esta campaña puede englobarse dentro de las acciones que favorecen la idea neoliberal de sociedad.
} 
Tal proyecto, desde el inicio, fue de carácter comunitario. A pesar de ese hecho, el desarrollo del mismo se ha caracterizado por el surgimiento y agudización de conflictos entre los pobladores de la isla. Existe un alto grado de competencia por el manejo de los recursos de la empresa comunitaria. Al grado de que ha habido personas encarceladas, distanciamiento entre familiares, violencia física, etcétera. Lo más paradójico es que es una isla poblada por aproximadamente veinte familias que se conocen de toda la vida (Ortiz, 2010 y 2013b).

Casos como el de la Isla de Yunuén dan cuenta de cómo la promoción de proyectos basados en la idea del fortalecimiento del capital social comunitario puede derivar precisamente en lo contrario. De hecho, se puede destruir el capital social previamente existente, ya que algunas instituciones tradicionales, como la del tequio, ${ }^{14}$ se han debilitado a partir de la entrada de diferentes programas de gobierno. ${ }^{15}$

Haciendo un análisis en perspectiva, las contradicciones antes expuestas nos llevan a dos posibles explicaciones. La primera se deriva de la funcionalidad que tiene la fragmentación de una comunidad, ya que eso debilita también su capacidad de acción política colectiva. Al no estar lo suficientemente cohesionados, es más fácil que los diferentes gobiernos puedan tener un mejor manejo de los potenciales conflictos político-sociales. En este sentido podría interpretarse como una estrategia de gobernabilidad. La segunda explicación es en relación a la promoción del capital social como elemento importante de la CAD. Si se requiere de ciudadanos organizados y cohesionados en función de la búsqueda de su propio desarrollo (como se plantea desde el modelo neoliberal), los diseñadores de políticas públicas enfocadas en la promoción de tales

\footnotetext{
${ }^{14}$ Se refiere al trabajo que prestan los miembros de la comunidad de forma gratuita, ya sea para la propia comunidad o para otras personas o familias de la misma. De esta forma se ayudan unos a otros en diferentes tareas que requieren de un esfuerzo colectivo. Ello refuerza los lazos de cooperación recíproca.

${ }^{15}$ Por ejemplo, se dice que desde la entrada del Programa Nacional de Solidaridad (Pronasol en la administración de Carlos Salinas, 1988-1994) algunas personas en la zona purépecha (Michoacán) ya no quieren participar en el tequio. Esto se debe a que el Pronasol introdujo la modalidad de recompensar con bienes materiales el trabajo para la comunidad, por lo que algunos miembros de la misma ya no quieren trabajar «gratis» (Ortiz, 2010).
} 
capacidades están pasando por alto la complejidad de los grupos sociales. Ello, sin lugar a dudas, es resultado de que parten de una idea simplista sobre el capital social (tipo Putnam). Al no considerar que existe la estratificación aun al interior de los grupos, dejan de lado el tema de la potencialidad de conflicto, lo que finalmente tiene como resultado el obtener lo contrario de lo que se proponían (debilitamiento del capital social comunitario).

Finalmente, es importante señalar que cualquier enfoque sobre el capital social que deje de lado el análisis de las condiciones estructurales estaría cayendo en una simplicidad peligrosa tanto en el sentido de su aplicación práctica (mediante el uso como categoría articuladora de política pública), como en el sentido académico (mediante el uso como categoría de análisis social). Sólo perspectivas como la de Bourdieu nos pueden ofrecer herramientas de análisis que dan cuenta de la complejidad de la dinámica de las relaciones sociales y de la reproducción de las diferencias de clase, la estratificación social, etcétera. Nos permite abordar la articulación de las dimensiones micro y macro de la dinámica social. Si se continúa empleando una visión como la de Putnam en el diseño de política pública, la única conclusión a la que podemos llegar es que se debe a su funcionalidad para la propuesta neoliberal de desarrollo. Sin embargo, habría que advertir que - como en el ejemplo arriba mencionado-, este uso puede ser perjudicial, incluso, para los propios fines de dicha modalidad de desarrollo.

\section{BIBLIOGRAFÍA}

Assies, Willem (1999), «Pueblos indígenas y reforma del Estado en América Latina», en Assies, van der Haar y Hoekema, El reto de la diversidad, Zamora, El Colegio de Michoacán. , Marco A. Calderón y Ton Salman (eds.) (2001), Ciudadanía, cultura política y reforma del Estado en América Latina, Zamora, El Colegio de Michoacán. 
Bevort, Antoine (2007), El capital Social y las teorías sociológicas. Breve historia intelectual del capital social, en http://www.eustat.es/document/datos/ CV07_02.pdf (5/06/2009)

Bourdieu, Pierre y J.D. Loïc Wacquant (1992), Respuestas por una antropología reflexiva, México, Grijalbo.

Durston, John (2002), El capital social campesino en la gestión del desarrollo rural. Díadas, equipos, puentes y escaleras, Santiago de Chile, CEPal.

Ezcurra, Ana María (1998), ¿Qué es el neoliberalismo? Evolución y límites de un modelo excluyente, Buenos Aires, Lugar Editorial, IDEAS.

Giménez, Gilberto (2002). «Introducción a la sociología de Pierre Bourdieu», Colección Pedagógica Universitaria, núm. 37-38.

Grimson, Alejandro (comp.) (2007), Cultura y neoliberalismo, Buenos Aires, Consejo Latinoamericano de Ciencias Sociales.

Hale, Charles (2003), «Does Multiculturalism Menace? Governance, cultural rigths and the politics of identity in Guatemala», Journal Latino America Studies, núm. 34.

KLIKSBerg, Bernardo (1999), «Capital social y cultura, claves esenciales del desarrollo», Revista de la CEPAL, núm. 69.

Millán, René y Sara Gordon (2004), «Capital Social: una lectura de tres perspectivas clásicas», Revista Mexicana de Sociología, núm. 4.

Montufar, César (2001), Hacia un nuevo marco interpretativo de la asistencia internacional para el desarrollo, en http://168.96.200.17/ar/libros/relint/ montufar.pdf (7/09/2005).

Ortiz Gómez, María Guadalupe (2010), Neoliberalismo de Sur a Norte en América Latina: la cultura de la autogestión para el desarrollo en comunidades indígenas, los casos de Chile y México, Tesis de doctorado en Ciencias Sociales, especialidad en Estudios Rurales, Zamora, El Colegio de Michoacán.

(2013a), «¿Neoliberalismo autogestivo? La cultura de autogestión para el desarrollo como herramienta analítica», Contextualizaciones Latinoamericanas, núm. 9. Disponible en: http://www.contextualizacioneslatinoamericanas. com.mx/pdf/NeoliberalismoautogestivoLaculturadeautogesti\%C3\%B3npar aeldesarrollocomoherramientaanal\%C3\%ADtica_9.pdf.

(2013b), «Yunuén, una comunidad que se desdibuja. Turismo y capital social en una isla del Lago de Pátzcuaro», Revista de Investigación Social, núm. 14.

Paley, Julia (2001), La «participación» y la «sociedad civil», Chile: Discursos internacionales, estrategias gubernamentales, respuestas organizacionales, ponencia 
presentada en el Congreso Latin American Studies Association (Lasa 2001), Washington, D.C., 6 al 8 de septiembre.

Portes, Alejandro (1998), «Social Capital: Its Origins and Applications in Modern Sociology», Annual Review of Sociology, núm. 24.

Putnam, Robert D. y Kristin A. Gross (2003), «Introducción», en Putnam, Robert D. (ed.), El declive del capital social. Un estudio internacional sobre las sociedades y el sentido comunitario, Barcelona, Galaxia Gutenberg, Círculo de Lectores.

Ramírez Plascencia, Jorge (2005), «Tres visiones del capital social: Bourdieu, Coleman y Putnam, Acta Republicana Politica y Sociedad, núm. 4. 